\title{
Outcomes of non-invasive diagnostic modalities for the detection of coronary artery disease: network meta-analysis of diagnostic randomised controlled trials
}

\author{
George CM Siontis, ${ }^{1}$ Dimitris Mavridis, ${ }^{2}$ John P Greenwood, ${ }^{3}$ Bernadette Coles, ${ }^{4}$ \\ Adriani Nikolakopoulou, ${ }^{5}$ Peter Jüni, ${ }^{6}$ Georgia Salanti, ${ }^{5}$ Stephan Windecker ${ }^{1}$
}

${ }^{1}$ Department of Cardiology,

Bern University Hospital,

Inselspital, Bern, Switzerland

${ }^{2}$ Department of Primary

Education, University of

Ioannina, loannina, Greece

${ }^{3}$ Leeds Institute of Cardiovascular and Metabolic Medicine, University of Leeds, Leeds, UK

${ }^{4}$ Cancer Research Wales Library, Velindre National Health Trust,

Cardiff, UK

${ }^{5}$ Institute of Social and

Preventive Medicine, University of Bern, Bern, Switzerland

${ }^{6}$ Applied Health Research Centre, Li Ka Shing Knowledge Institute, St Michael's Hospital, University of Toronto, Toronto, Canada

Correspondence to: SWindecker stephan.windecker@insel.ch Additional material is published online only. To view please visit the journal online.

Cite this as: $B M J$ 2018;360:k452 http://dx.doi.org/10.1136/bmi.k452

Accepted: 16 January 2018

\section{ABSTRACT}

OBJECTIVE

To evaluate differences in downstream testing, coronary revascularisation, and clinical outcomes following non-invasive diagnostic modalities used to detect coronary artery disease.

\section{DESIGN}

Systematic review and network meta-analysis.

\section{DATA SOURCES}

Medline, Medline in process, Embase, Cochrane Library for clinical trials, PubMed, Web of Science, SCOPUS, WHO International Clinical Trials Registry Platform, and Clinicaltrials.gov.

\section{ELIGIBILITY CRITERIA FOR SELECTING STUDIES}

Diagnostic randomised controlled trials comparing non-invasive diagnostic modalities in patients presenting with symptoms suggestive of low risk acute coronary syndrome or stable coronary artery disease.

\section{DATA SYNTHESIS}

A random effects network meta-analysis synthesised available evidence from trials evaluating the effect of non-invasive diagnostic modalities

\section{WHAT IS ALREADY KNOWN ON THIS TOPIC}

Information on diagnostic accuracy is important for decisions on the usefulness of a diagnostic test, which might not translate into patient benefits

Diagnostic randomised controlled trials provide the most conclusive evidence regarding patient outcomes, and represent a rigorous approach to diagnostic test evaluation

Several non-invasive imaging modalities can be used to investigate patients with suspected low risk acute coronary syndromes or stable coronary artery disease, but their effect on downstream testing and clinical outcomes remains unknown and inconsistent

\section{WHAT THIS STUDY ADDS}

In patients with low risk acute coronary syndrome, functional testing (stress echocardiography and cardiovascular magnetic resonance) is associated with fewer referrals for downstream invasive coronary angiography than coronary computed tomographic angiography, without apparent impact on subsequent risk of myocardial infarction

Among patients with suspected stable coronary artery disease, functional testing (stress echocardiography and single photon emission computed tomographymyocardial perfusion imaging) is associated with less requirement for additional diagnostic investigations (overall downstream testing) than coronary computed tomographic angiography, although the estimates cannot rule out a significant effect on the risk of myocardial infarction associated with individual tests Future adequately powered clinical trials should evaluate more broadly defined clinical outcomes, subsequent use of hospital resources, and cost effectiveness aspects of implemented strategies, which are representative of current clinical practice

on downstream testing and patient oriented outcomes in patients with suspected coronary artery disease. Modalities included exercise electrocardiograms, stress echocardiography, single photon emission computed tomographymyocardial perfusion imaging, real time myocardial contrast echocardiography, coronary computed tomographic angiography, and cardiovascular magnetic resonance. Unpublished outcome data were obtained from 11 trials.

\section{RESULTS}

18 trials of patients with low risk acute coronary syndrome $(n=11329)$ and 12 trials of those with suspected stable coronary artery disease $(n=22062)$ were included. Among patients with low risk acute coronary syndrome, stress echocardiography, cardiovascular magnetic resonance, and exercise electrocardiograms resulted in fewer invasive referrals for coronary angiography than coronary computed tomographic angiography (odds ratio 0.28 (95\% confidence interval 0.14 to 0.57 ), 0.32 ( 0.15 to 0.71 ), and 0.53 ( 0.28 to 1.00 ), respectively). There was no effect on the subsequent risk of myocardial infarction, but estimates were imprecise. Heterogeneity and inconsistency were low. In patients with suspected stable coronary artery disease, an initial diagnostic strategy of stress echocardiography or single photon emission computed tomographymyocardial perfusion imaging resulted in fewer downstream tests than coronary computed tomographic angiography (0.24 (0.08 to 0.74$)$ and 0.57 ( 0.37 to 0.87 ), respectively). However, exercise electrocardiograms yielded the highest downstream testing rate. Estimates for death and myocardial infarction were imprecise without clear discrimination between strategies.

\section{CONCLUSIONS}

For patients with low risk acute coronary syndrome, an initial diagnostic strategy of stress echocardiography or cardiovascular magnetic resonance is associated with fewer referrals for invasive coronary angiography and revascularisation procedures than non-invasive anatomical testing, without apparent impact on the future risk of myocardial infarction. For suspected stable coronary artery disease, there was no clear discrimination between diagnostic strategies regarding the subsequent need for invasive coronary angiography, and differences in the risk of myocardial infarction cannot be ruled out. 


\section{Introduction}

Chest pain is a leading cause for physician consultation that leads to several million office and emergency department visits as well as hospital admissions yearly. ${ }^{12}$ Despite the use of clinical decision rules ${ }^{3-5}$ and the improved sensitivity of cardiac biomarkers, ${ }^{6-8}$ many patients who are admitted to the emergency department in order to exclude an acute coronary syndrome are ultimately found not to have a cardiac cause of their symptoms. Conversely, patients with symptoms who undergo cardiac investigations because of chest pain and are diagnosed with non-cardiac causes or in whom the cause of chest pain remains undetermined are at increased risk of subsequent cardiovascular events and death. ${ }^{910}$

Guidelines published in 2012 by the American College of Cardiology Foundation/American Heart Association recommended the use of functional testing, mainly on the basis of evidence derived from studies of diagnostic accuracy (because the vast majority of diagnostic randomised trials were published after these guidelines). ${ }^{11}$ However, recent audits in large numbers of patients showed only a modest effect on subsequent diagnostic findings. ${ }^{12}$ Currently, functional and anatomical non-invasive tests are widely available and used according to locally available resources and expertise (box): exercise electrocardiograms, single photon emission computed tomography (CT)-myocardial perfusion imaging, stress echocardiography, real time myocardial contrast echocardiography, coronary
CT angiography, and cardiovascular magnetic resonance. Among these, coronary CT angiography as the only non-invasive anatomical diagnostic modality has been suggested to overcome limitations of traditional functional testing and has undergone close scrutiny in recent years. ${ }^{13} 14$ So far, diagnostic randomised controlled trials do not provide conclusive evidence as to whether a non-invasive anatomical or functional testing strategy gives the best results for subsequent downstream testing or clinical outcomes. Therefore, we summarised the available evidence and evaluated clinical endpoints of different non-invasive diagnostic modalities in patients with symptoms suggestive of coronary artery disease through network meta-analysis.

\section{Methods}

The detailed protocol that follows the template of a Cochrane review for multiple interventions is available in PROSPERO registry (CRD42016049442), ${ }^{15}$ and was prepared according to the guidelines of the Cochrane Multiple Interventions Methods Group. ${ }^{16}$

\section{Data sources and searches}

We performed a broad literature search in Medline, Medline in process, Embase, Cochrane Library for clinical trials, PubMed, Web of Science, SCOPUS, WHO International Clinical Trials Registry Platform, and Clinicaltrials.gov (appendix 1). A search algorithm was developed and adapted for each database, without language or sample size restrictions.

\section{Box: Key features of widely used functional and anatomical tests for non-invasive diagnosis of coronary artery disease}

\section{Exercise electrocardiograms}

This test aims to detect myocardial ischaemia indirectly through electrocardiographic changes during exercise and recovery, which is the physiological consequence of a mismatch between myocardial oxygen supply (coronary blood flow) and myocardial oxygen demand (myocardial work). It is a well validated tool for the assessment of functional capacity and chronotropic response to exercise.

\section{Stress echocardiography}

Cardiac ultrasound (echocardiography) is used to evaluate myocardial function (contractility) at rest, and during exercise or pharmacological stress. It can detect the presence and extent of coronary artery disease by provoking regional ischaemia with resulting wall motion abnormalities. Myocardial ischaemia is provoked either by exercise (treadmill or bicycle) or pharmacological agents (predominantly dobutamine).

\section{Real time myocardial contrast echocardiography}

This test uses an intravenous echocardiographic contrast agent during stress echocardiography. While echo-contrast agents can be used to improve endocardial border definition in patients with suboptimal echocardiographic images, they also allow visualisation of myocardial tissue perfusion.

\section{Single photon emission computed tomography-myocardial perfusion imaging}

This technique uses intravenous administration of a radioactive myocardial perfusion tracer (radioisotope) to evaluate cardiac perfusion and function at rest and during dynamic exercise or pharmacological stress. The technique provides information on the presence or absence of myocardial ischaemia, myocardial infarction (and viability), and ventricular function.

\section{Coronary computed tomographic angiography}

This test allows direct visualisation of the coronary artery lumen and wall using an intravenous contrast agent to produce a computed tomographic coronary angiogram. Preceding non-contrast scans can assess the presence and extent of coronary artery calcium in the vessel wall, which is a marker of extent of coronary atherosclerosis and future risk, but not necessarily related to the severity of coronary artery narrowing.

\section{Stress cardiovascular magnetic resonance imaging}

This advanced cross sectional imaging procedure acquires two or three dimensional images of the heart. Using a contrast agent during pharmacological stress, first pass perfusion images can be used to identify areas of low myocardial blood flow (ischaemia) or stress induced regional wall motion abnormalities. During a single study, information is also provided on regional or global resting ventricular function, myocardial infarction (and viability), and proximal coronary artery anatomy. 


\section{Study selection}

We included diagnostic randomised controlled trials comparing any non-invasive anatomical (evaluation of coronary anatomy) or functional (detection of myocardial ischaemia) diagnostic strategy for the detection of coronary artery disease in patients without previously known coronary artery disease but presenting with symptoms suggestive of low risk acute coronary syndrome or stable coronary artery disease. Low risk acute coronary syndrome was defined as patients typically presenting with chest pain (or anginal equivalent) for at least 5 minutes at rest within the past 24 hours, without history of known coronary artery disease, without diagnostic ischaemic changes on electrocardiogram, without haemodynamic or clinical instability, and an initial troponin level lower than the 99th percentile of the used assay. These patients typically do not need immediate assessment by invasive coronary angiography.

We also considered trials comparing any diagnostic strategy with a standard of care as defined by the authors, and trials that allowed in the same arm any diagnostic test of the same testing group (that is, functional testing). We included trials in which at least one comparative pair non-invasive diagnostic tests was available, irrespective of the number of trial arms. As a condition, we consider that within each patient group, any of the diagnostic approaches could have been applied and hence they can be considered "jointly randomiseable." 17

\section{Data extraction and quality assessment}

Characteristics of trials, patients, and diagnostic strategies, were summarised. Details of the extracted items are available in the online protocol. Following the index diagnostic strategy, we considered as primary endpoints the subsequent referral to invasive coronary angiography and any coronary revascularisation. We considered the rate of invasive coronary angiography as an indicator of downstream testing, and the rate of revascularisation as surrogate for clinically significant coronary artery disease. Downstream testing is the need for additional diagnostic investigations that are performed (invasive or non-invasive (or both)) after the initial diagnostic test or strategy. Typically, this might occur after test failure or diagnostic uncertainty in relation to the index test result.

The outcome parameters were chosen on the basis of the hypothesis that a non-invasive, anatomically driven strategy might be more sensitive to identify non-clinically significant coronary artery disease (as suggested by previous studies), which in turn could have a prognostic effect. ${ }^{18} 19$ We also collected information on patient oriented outcomes (myocardial infarction and death), and overall downstream testing (including the number of additional diagnostic tests after the index diagnostic intervention during followup). In case of missing outcomes in the main and subsequent publications of the included trials, we contacted the principal investigator of each trial to provide additionally required information.
We evaluated the internal validity of the trials by using the Cochrane risk of bias tool. ${ }^{20}$ Each item was described as being at low, high, or unclear risk of bias for random sequence generation, allocation concealment, blinding (participants/personnel and outcomes), incomplete outcome data, and selective outcome reporting. We evaluated the risk of bias in each trial and pairwise comparison overall for the outcome of referral to invasive coronary angiography as low, moderate, or high risk of bias, based on our judgments for allocation concealment and blinding of outcome assessment.

\section{Data synthesis and analysis}

We performed the predefined analyses separately for each group of study population as defined according to clinical presentation, and for each outcome according to established meta-analytical methods. ${ }^{21} 22$ The detailed analysis plan is available in PROSPERO (CRD42016049442). Study specific odds ratios were synthesised by use of random effects pairwise and network meta-analysis. ${ }^{23-27}$ A treatment hierarchy was obtained according to ranking probabilities summarised with the surface under the cumulative ranking curve. ${ }^{28}$ Weestimated a common heterogeneity by using restricted maximum likelihood and evaluated its magnitude by comparing the estimated variance $\left(\tau^{2}\right)$ to its empirical distribution for pharmacological interventions and semiobjective outcomes. According to the empirical distribution, the median is 0.04 (interquartile range $0-1.58) .{ }^{29}$ Estimates around 0.04, 0.16, and 0.36 can be considered to represent a low, moderate, and high degree of heterogeneity, respectively. ${ }^{30}$ To evaluate the assumption of consistency, ${ }^{31}$ we compared the direct and indirect evidence within each loop of evidence ${ }^{32}$ and used a design by treatment test. ${ }^{33}$ In case of important heterogeneity or inconsistency, we planned to explore sources using potential effect modifiers as described in the protocol, but this was not possible owing to the low number of trials.

In primary analyses, we considered each individual diagnostic strategy separately as applied in each trial. Two trials, CRESCENT ${ }^{34}$ and PROMISE, ${ }^{35}$ randomised patients to an anatomical versus a functional diagnostic strategy using functional tests that are widely available in clinical practice (exercise electrocardiograms, stress echocardiography, and single photon emission CT-myocardial perfusion imaging). For the primary analysis, these trials were assigned as having randomised the patients to exercise electrocardiograms ${ }^{34}$ and to single photon emission CT-myocardial perfusion imaging, ${ }^{35}$ which were the most frequently used tests in each trial.

In secondary analyses, we grouped traditional functional tests in a single node and compared them with coronary CT angiography as a purely anatomical test and cardiovascular magnetic resonance as an advanced imaging modality. Cardiovascular magnetic resonance was not included in the node of functional tests because of the different nature and availability of this advance imaging modality. This merging of tests 


\section{A. Low risk acute coronary syndrome}

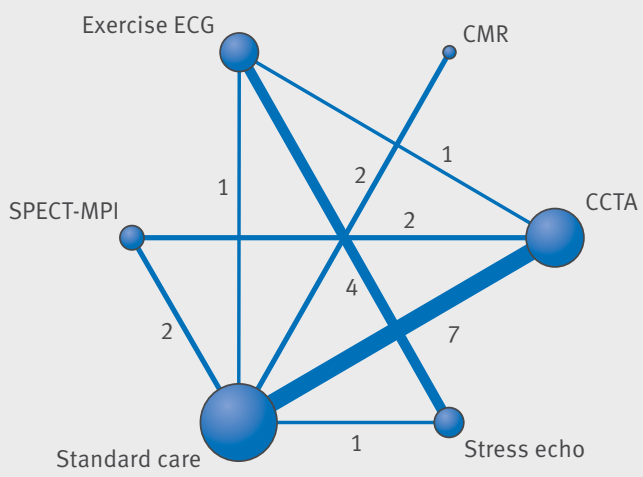

C. Stable coronary artery disease

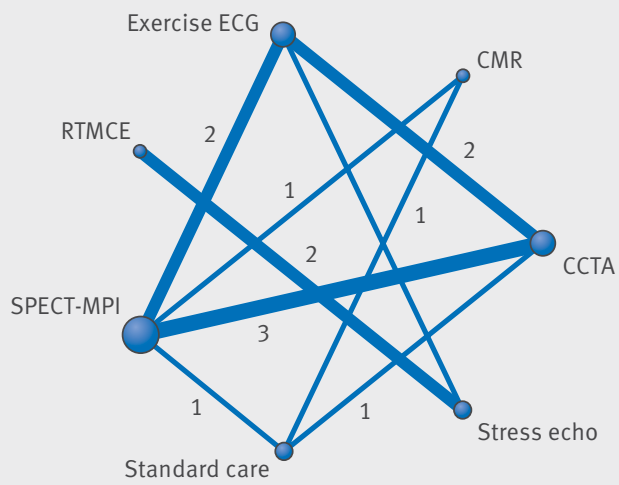

B. Low risk acute coronary syndrome functional testing grouped

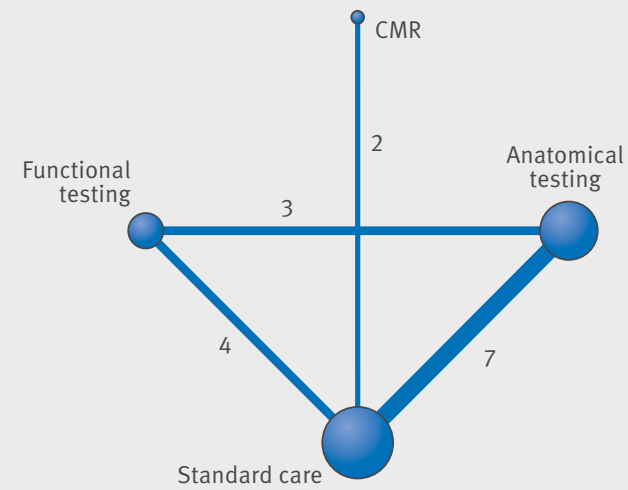

D. Stable coronary artery disease functional testing grouped

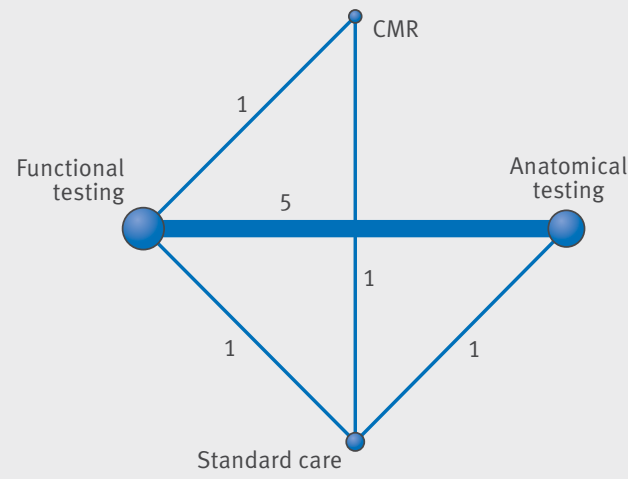

Fig 1 | Network plots of examined diagnostic strategies across different patient groups. Network plots show comparisons across the available diagnostic strategies for each group of study populations ( $A$ and $C$ ), and consider stress echocardiography, single photon emission computed tomography-myocardial perfusion imaging, exercise electrocardiograms, or real time myocardial contrast echocardiography in the same group of diagnostic modalities of traditional functional testing ( $B$ and $D$ ). Anatomical testing pertains to coronary computed tomographic angiography.

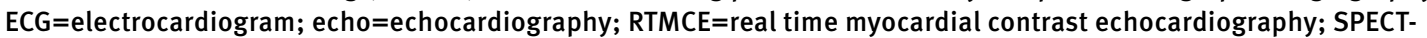
$\mathrm{MPI}=$ single photon emission computed tomography-myocardial perfusion imaging; CCTA=coronary computed tomographic angiography; $C M R=$ cardiovascular magnetic resonance

is expected to increase the power of the analysis. ${ }^{36}$ Finally, we did a sensitivity analysis by excluding the two trials ${ }^{3435}$ that did not randomise the patients to an individual diagnostic strategy.

We calculated numbers needed to treat and numbers needed to harm for each outcome and each group of patients after grouping for functional testing as mentioned above, by applying the estimated odds ratios and confidence intervals to the odds of events estimated in patients randomly allocated to an anatomical based strategy (coronary CT angiography). For this purpose, we considered a baseline risk based on the event rates in the ACRIN-PA ${ }^{37} 38$ and PROMISE ${ }^{35}$ trials for patients presenting with low risk acute coronary syndrome and suspected of stable coronary artery disease, respectively. Both trials were the largest in each group of patients (low risk acute coronary syndrome and stable coronary artery disease) and examined the role of an anatomical testing strategy.

Analyses were performed in Stata (network and network graph packages). ${ }^{39}$ To illustrate the assessments of risk of bias for the primary outcome of referral to invasive coronary angiography, we produced a bar graph showing how much information came from high, moderate, and low risk of bias trials for each network estimate by using CINeMA (Confidence in Network Meta-Analysis) software. ${ }^{40}$ We assessed the effect of study limitations in all network meta-analysis estimates using graphs based on the contribution matrix. $^{31}$

\section{Patient involvement}

No patients were involved in the development of the research question, development of outcome measures, design, or conduct of this study. No patients were asked to advise on interpretation or writing up of results. There are no plans to involve patients in the dissemination of the results of this study.

\section{Results}

Our search yielded 19674 citations, which were initially screened on abstract level for eligibility; 101 

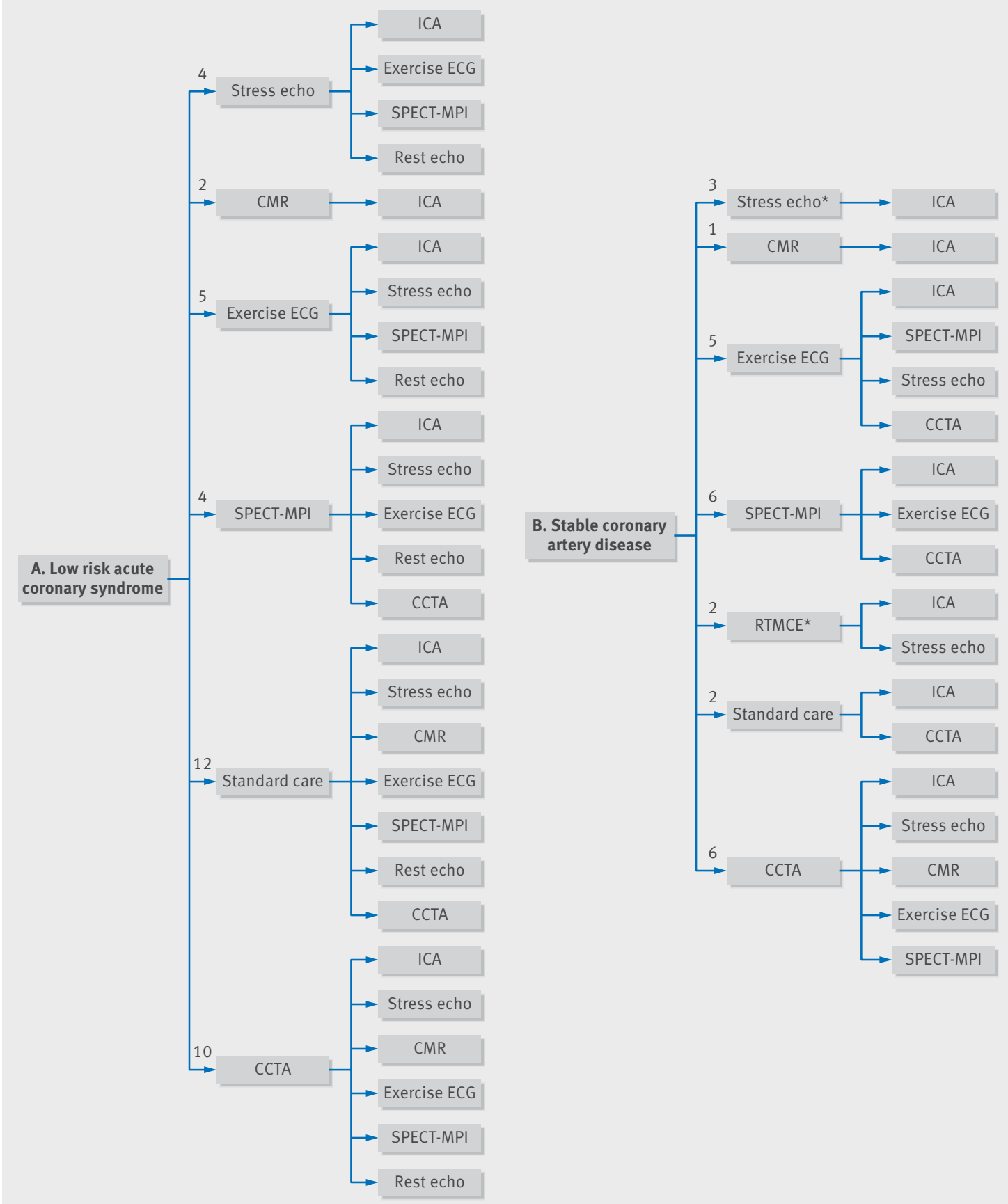

Fig 2 | Pathways taken following the index diagnostic intervention across included diagnostic randomised controlled trials for patients with (A) low risk acute coronary syndrome and (B) suspected stable coronary artery disease.

Numbers indicate total number of trials in which each index diagnostic strategy (arm of randomisation) was applied. The final list of diagnostic strategies in each graph refer to downstream tests performed after the index diagnostic strategy. *Information on downstream testing is missing from one trial. ICA=invasive coronary angiography; ECG=electrocardiogram; echo=echocardiography; RTMCE=real time myocardial contrast echocardiography; SPECT$\mathrm{MPI}=$ single photon emission computed tomography-myocardial perfusion imaging; $C \mathrm{CTA}=$ coronary computed tomographic angiography; $C M R=$ cardiovascular magnetic resonance

reports were retrieved and reviewed in full text. Finally, 30 diagnostic randomised controlled trials (34 reports) including 33391 patients (16083 women) and six different imaging modalities were deemed eligible (box, fig 1, appendices 1 and 2). Appendix 3 shows the diagnostic accuracy of the evaluated imaging modalities based on previously published studies (appendix 4 lists abbreviations used). Figure 2 shows the diagnostic pathways chosen following the index diagnostic intervention across the trials. Descriptive details of the included trials and resulting networks are provided in table 1, figure 1, and appendix 5 . 
Table 1 | Diagnostic randomised controlled trials and characteristics

\begin{tabular}{|c|c|c|c|c|c|}
\hline $\begin{array}{l}\text { Trial first author, } \\
\text { publication year }\end{array}$ & Recruitment period & Source of funding & $\begin{array}{l}\text { No of } \\
\text { centres }\end{array}$ & Comparisons & $\begin{array}{l}\text { Sample } \\
\text { size }\end{array}$ \\
\hline BEACON, ${ }^{41} 2016$ & July 2011-January 2014 & Non-industry & 7 & CCTA $v$ standard care & 500 \\
\hline CT-COMPARE, ${ }^{43} 2014$ & March 2010-April 2011 & Non-industry & 1 & CCTA $v$ exercise ECG & 562 \\
\hline CATCH, ${ }^{4445} 2013$ & January 2010-January 2013 & Industry related & 1 & CCTA $v$ standard care & 600 \\
\hline Lim et al, ${ }^{46} 2013$ & August 2000-May 2002 & Non-industry & 1 & SPECT-MPI $v$ standard care & 1690 \\
\hline Miller et $\mathrm{al}^{47} 2013$ & 2011 & Non-industry & 1 & CMR $v$ standard care & 105 \\
\hline CT-STAT, ${ }^{49} 2011$ & June 2007-November 2008 & Industry related & 16 & CCTA $v$ SPECT-MPI & 749 \\
\hline Miller et al, ${ }^{50} 2011$ & October 2008-February 2009 & Non-industry & 1 & CCTA $v$ standard care & 60 \\
\hline Miller et al, ${ }^{5152} 2010$ & January 2008-April 2009 & Non-industry & 1 & CMR $v$ standard care & 109 \\
\hline Nucifora et al, ${ }^{53} 2009$ & Not reported & None reported & 10 & Stress echo $v$ exercise ECG $v$ standard care & 290 \\
\hline Chang et $\mathrm{al}^{54} 2008$ & May 2006-February 2007 & None reported & 1 & CCTA $v$ standard care & 266 \\
\hline Goldstein et al,,$^{55} 2007$ & March 2005-September 2005 & Industry related & 1 & CCTA $v$ standard care & 197 \\
\hline \multicolumn{6}{|c|}{ Stable coronary artery disease } \\
\hline IAEA-SPECT/CTA, ${ }^{60} 2016$ & June 2011-June 2014 & Non-industry & 6 & CCTA $\vee$ SPECT-MPI & 303 \\
\hline CE-MARC $2,{ }^{61} 2016$ & November 2012-March 2015 & Non-industry & 6 & CMR $v$ SPECT-MPI $v$ standard care & 1202 \\
\hline CRESCENT, $^{34} 2016$ & April 2011-July 2013 & Non-industry & 4 & CCTA $v$ functional testing ${ }^{\star}$ & 350 \\
\hline Zacharias et al, ${ }^{62} 2016$ & February 2013-March 2014 & None reported & 1 & Stress echo $v$ exercise ECG & 385 \\
\hline PROMISE, ${ }^{35} 2015$ & July 2010-September 2013 & Non-industry & 193 & CCTA $v$ functional testing ${ }^{\star}$ & 10003 \\
\hline SCOT-HEART, ${ }^{6364} 2015$ & November 2010-September 2014 & Non-industry & 12 & CCTA $v$ standard care & 4146 \\
\hline Laiq et al,$^{65} 2015$ & October 2007-October 2011 & Industry related & 1 & RTMCE $v$ stress echo & 1649 \\
\hline CAPP, $^{66} 2015$ & September 2010-November 2011 & Non-industry & 2 & CCTA $v$ exercise ECG & 500 \\
\hline Porter et al, ${ }^{67} 2013$ & October 2007-December 2011 & Non-industry & 1 & RTMCE $v$ stress echo & 2063 \\
\hline Min et al, ${ }^{68} 2012$ & December 2008-June 2009 & Industry related & 2 & CCTA $\vee$ SPECT-MPI & 180 \\
\hline WOMEN, ${ }^{69} 2011$ & Not reported & Industry related & 43 & SPECT-MPI $v$ exercise ECG & 824 \\
\hline Sabharwal et al, ${ }^{70} 2007$ & February 2001-July 2002 & Industry related & 1 & SPECT-MPI $v$ exercise ECG & 457 \\
\hline
\end{tabular}

Trials ordered chronologically, starting from most recently published trial. ECG=electrocardiogram; echo=echocardiography; RTMCE=real time myocardial contrast echocardiography; SPECT-MPI=single photon emission computed tomography-myocardial perfusion imaging; CCTA=coronary computed tomographic angiography; $C$ MR=cardiovascular magnetic resonance. *Functional testing includes exercise electrocardiograms, stress echocardiography, or single photon emission computed tomography-myocardial perfusion imaging, as has been defined in individual trials.

Eligible trials included patients with low risk acute coronary syndrome (18 trials, 11329 patients) $)^{37} 38$ 41-59 and suspected stable coronary artery disease (12 trials, 22062 patients $^{34}{ }^{35}{ }^{60-70}$; table 1 ). The recruitment period for most of the trials was completed during the last 10 years. Seven trials reported industry related funding, 17 obtained exclusively non-industry funding, and six disclosed no funding. Fifteen trials were multicentre and two included three arm comparisons. In only three ${ }^{41436}$ trials of patients with low risk acute coronary syndrome, the authors clarified the use of a high sensitive troponin assay. A diagnostic strategy based on the recommended standard of care was included in 11 trials on acute coronary syndrome and two on stable coronary artery disease. Appendix 5 provides event rates for each assessed outcome parameter. We obtained unpublished outcome data from 11 trials $^{3435414244565761-6366}$ by contacting the principal investigators.

Most trials were considered to be at low risk in five of the assessed areas of potential bias, whereas no trial blinded the participants and personnel to the allocated diagnostic interventions (appendix 6). One $\operatorname{trial}^{43}$ was judged to be at high risk of bias related to random sequence generation and allocation concealment, one $^{44}$ was considered high risk in relation to the blinding of outcome assessment, and one ${ }^{51}$ had possible incomplete data reporting. The risk of bias was frequently unclear, owing to limited reporting in the publications. Appendix 6 details the results of the risk of bias assessment and the contribution of comparisons with low, moderate, and high risk of bias to each network meta-analysis estimate.

\section{Comparative efficacy of non-invasive diagnostic strategies}

Patients with low risk acute coronary syndrome

Patients initially evaluated by stress echocardiography, cardiovascular magnetic resonance, or exercise electrocardiograms to exclude coronary artery disease among patients with low risk acute coronary syndrome were less likely to be referred to invasive coronary angiography compared with coronary CT angiography (odds ratio 0.28 (95\% confidence 0.14 to 0.57 ), 0.32 (0.15 to 0.71 ), and 0.53 (0.28 to 1.00$)$, respectively). However, differences were marginal for single photon emission CT-myocardial perfusion imaging (0.78 (0.58 to 1.03)) and standard of care (0.85 (0.69 to 1.05)) compared with coronary CT angiography (fig 1A, fig 3). Heterogeneity was low ( $\tau^{2}=0.023$; fig 3 , appendix 7$)$. 


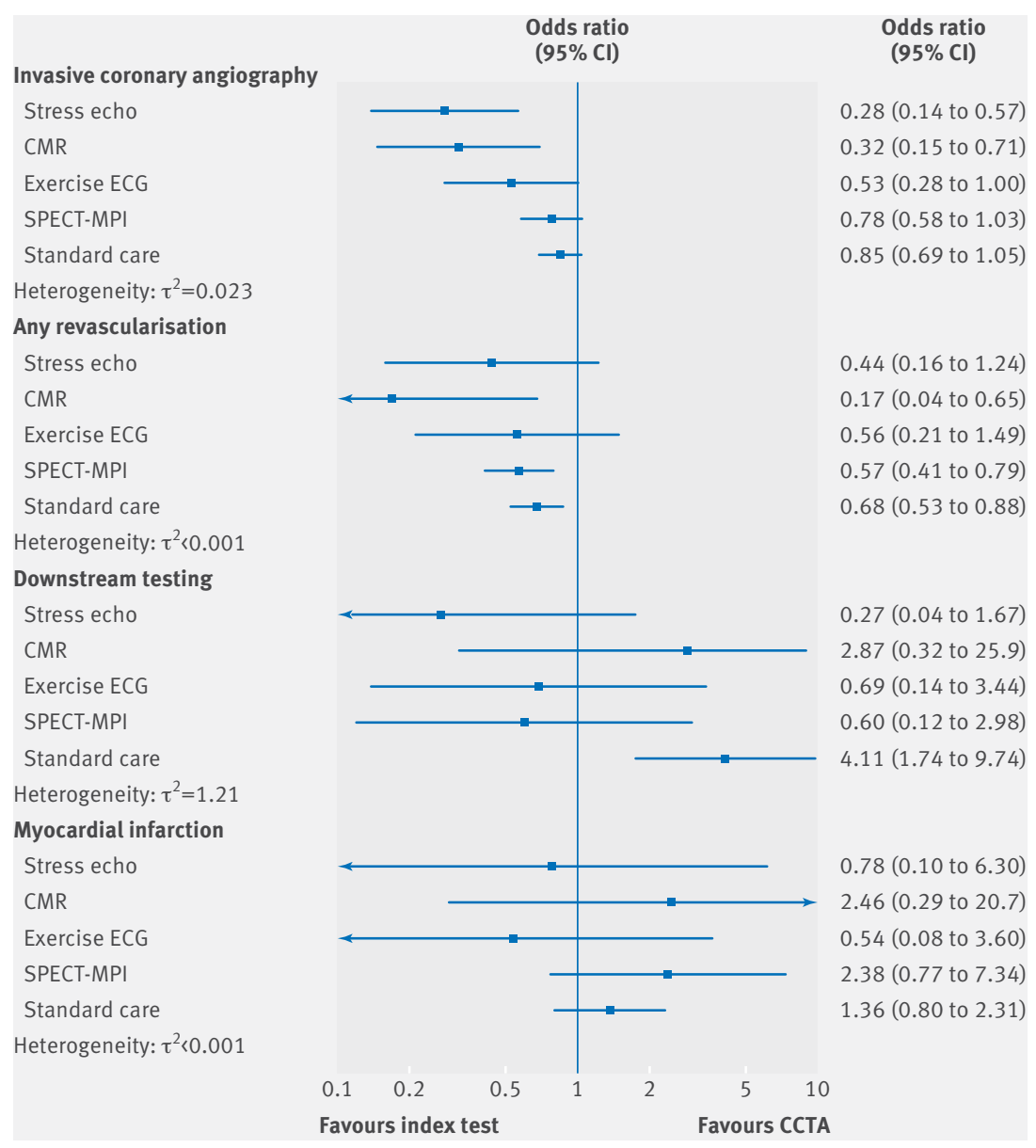

Fig 3 | Network meta-analysis effects of examined individual diagnostic strategies versus coronary computed tomographic angiography (anatomical testing), for study group of patients with low risk acute coronary syndrome. Forest plot considers individual diagnostic strategies, as shown in figure 1A. Index test indicates any diagnostic modality other than coronary computed tomographic angiography (anatomical testing). Network meta-analysis for the outcome of death was not feasible because of missing data and zero events. ECG=electrocardiogram; echo=echocardiography; SPECT-MPI=single photon emission computed tomographymyocardial perfusion imaging; $C \mathrm{CTA}=$ coronary computed tomographic angiography; $\mathrm{CMR}=$ cardiovascular magnetic resonance with consistent findings between direct and indirect evidence.

Despite the differences between the diagnostic modalities in referrals for invasive coronary angiography and revascularisation, none of the strategies affected the rate of subsequent myocardial infarction, although the derived estimates were not precise. In terms of overall downstream testing, all diagnostic strategies (apart from cardiovascular magnetic resonance) were statistically significant better than a standard of care approach, which resulted in the highest rate of downstream testing (appendix 7). Significant disagreement between direct and indirect estimates was not identified, but heterogeneity was high $\left(\tau^{2}=1.21\right)$.

After grouping of widely available functional tests (including exercise electrocardiograms, stress echocardiography, or single photon emission CTmyocardial perfusion imaging; fig 1B), a functional testing strategy and cardiovascular magnetic resonance were less likely than an anatomical testing (coronary CT angiography) strategy to lead to referrals for invasive coronary angiography (odds ratio 0.71 (95\% confidence interval 0.53 to 0.96$)$ and 0.32 (0.15 to 0.72 ), respectively; $\tau^{2}=0.032$; fig 4 , appendix 8). Considering overall downstream testing, a functional testing strategy was ranked first but without significant difference compared with an anatomical testing strategy. For both outcomes of downstream testing and myocardial infarction, the derived estimates were imprecise.

Table 2 presents the estimated numbers needed to treat to prevent one event and numbers needed to harm to cause one event for different outcomes after grouping of functional diagnostic strategies. For example, in the comparison of functional testing versus anatomical testing in terms of risk of myocardial infarction, the number needed to harm was 133 . The 95\% confidence interval indicated that results were compatible with both a beneficial effect of a strategy based on functional versus anatomical testing, resulting in a number needed to treat to prevent one event of 194 or more. The confidence interval also indicated that results were compatible with a harmful effect of functional testing, resulting in a number needed to harm to cause one event of 25 or more.

\section{Patients with suspected stable coronary artery disease}

Seven individual non-invasive diagnostic strategies were examined and constitute the network of patients with symptoms assessed for suspected stable coronary artery disease (fig 1C). For the outcomes of invasive coronary angiography referral and revascularisation, no individual diagnostic strategy differed from an anatomical strategy (coronary CT angiography), apart from single photon emission CT-myocardial perfusion imaging that marginally yielded less invasive coronary angiography (odds ratio 0.54 (95\% confidence interval 0.30 to 0.98$)$ ) and subsequent revascularisations (0.57 (0.37 to 0.87); fig 5). There was no evidence 


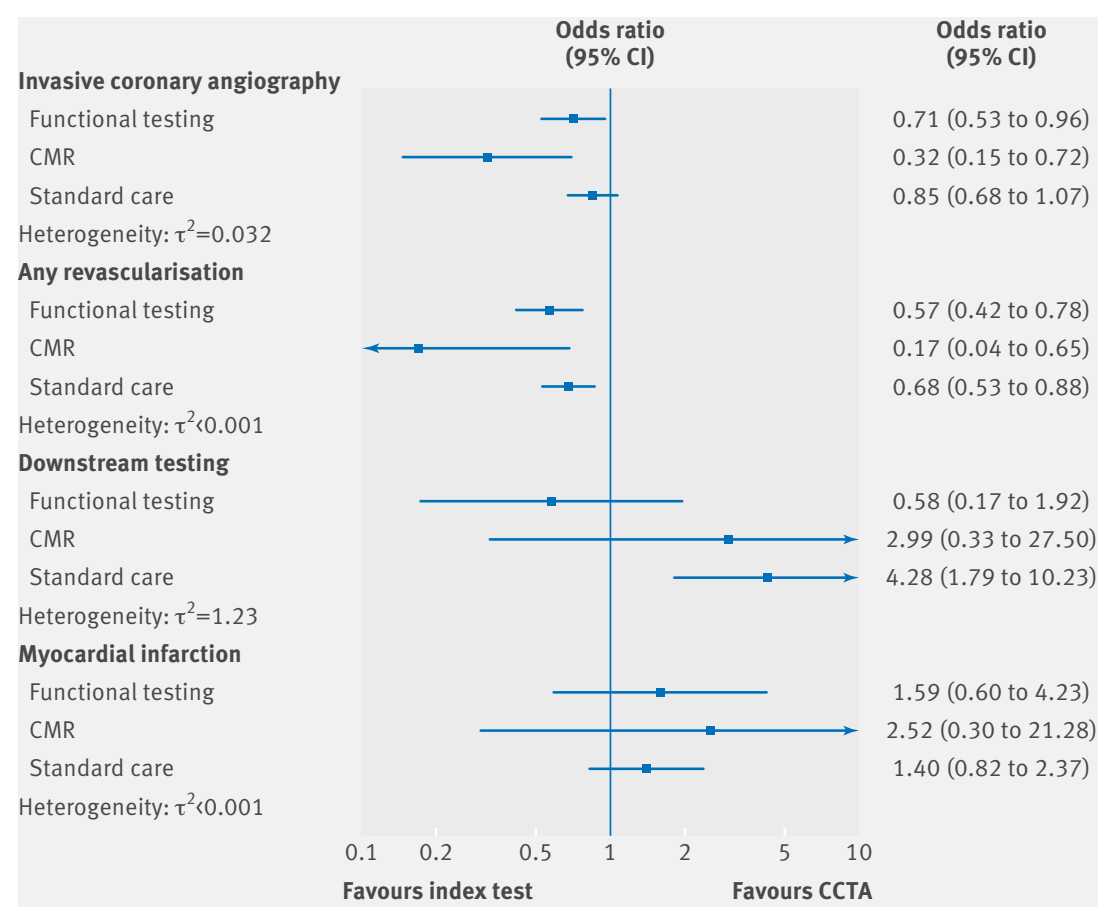

Fig 4 | Network meta-analysis effects of examined grouped diagnostic strategies versus coronary computed tomographic angiography (anatomical testing), for study group of patients with low risk acute coronary syndrome. Forest plot considers grouping of functional tests, as shown in figure 1B. Index test indicates any diagnostic modality other than coronary computed tomographic angiography (anatomical testing). Network meta-analysis for the outcome of death was not feasible because of missing data and zero events. $C C T A=$ coronary computed tomographic angiography; $C M R=$ cardiovascular magnetic resonance of inconsistency $(\mathrm{P}=0.62)$ and heterogeneity was moderate to high $\left(\tau^{2}=0.336\right)$ for referrals to invasive coronary angiography. Overall, downstream testing favoured an initial diagnostic strategy with stress echocardiography $(0.24(0.08$ to 0.74$))$ or single photon emission CT-myocardial perfusion imaging (0.57 (0.37 to 0.87)), but not cardiovascular magnetic resonance (0.95 (0.41 to 2.22)) compared with coronary CT angiography (fig 5, appendix 9). Exercise electrocardiograms were ranked last, with the highest rate of downstream testing compared with any other diagnostic strategy. The overall heterogeneity was moderate $\left(\tau^{2}=0.137\right)$.

We detected inconsistency in the loop of coronary CT angiography, exercise electrocardiograms, and single photon emission CT-myocardial perfusion imaging, which was also verified with the sidesplit approach ( $\mathrm{P}=0.48$ for the inconsistency model overall). In the main analysis, we did not obtain a clear discrimination for most of the individual diagnostic strategies (imprecise estimates with broad confidence intervals and visually flat rankograms) for the clinical outcomes of myocardial infarction and death (fig 5, appendix 9).

In pairwise meta-analysis, functional testing resulted in fewer referrals for invasive coronary angiography (odds ratio 0.65 (95\% confidence interval 0.58 to 0.74$), \tau^{2}<0.001$ ) than non-invasive anatomical testing (coronary CT angiography), with a similar estimate (0.63 (0.44 to 0.90)) from network meta-analysis after grouping of the functional tests (fig 1D). Cardiovascular magnetic resonance ranked first with regards to fewer referrals for invasive coronary angiography (with an estimate of 0.37 (0.19 to 0.72 )) compared with standard of care in indirect comparisons (appendix 10), although with detectable inconsistency $(\mathrm{P}<0.05$ in sidesplit approach). In the network of strategies, functional testing resulted in fewer revascularisation procedures than anatomical testing (0.57 (0.41 to 0.78 ); fig 6 ), and this finding was consistent between direct and indirect evidence with low heterogeneity $\left(\tau^{2}=0.029\right)$. Despite differences in referrals for invasive assessment and subsequent revascularisation, no statistical significant difference was observed for the outcome of myocardial infarction for patients who were initially evaluated with a functional or anatomical based strategy (appendix 10). However, there was a trend towards favouring an anatomical testing strategy (fig 6).

In a sensitivity analysis excluding two trials $\left(\right.$ CRESCENT $^{34}$ and PROMISE ${ }^{35}$ ) that randomised the patients to a non-specific functional test, the obtained estimates did not allow for any clear discrimination between the individual diagnostic

\begin{tabular}{|c|c|c|c|c|}
\hline & $\begin{array}{l}\text { Baseline } \\
\text { riskt }\end{array}$ & $\begin{array}{l}\text { Functional testing } v \\
\text { anatomical testing }\end{array}$ & $\begin{array}{l}\text { Cardiovascular magnetic resonance } v \\
\text { anatomical testing }\end{array}$ & $\begin{array}{l}\text { Standard care } v \\
\text { anatomical testing }\end{array}$ \\
\hline \multicolumn{5}{|l|}{ Low risk acute coronary syndromeł } \\
\hline Invasive coronary angiography* & 7.4 & NNT 49 (NNT 30 to NNT 364) & NNT 20 (NNT 16 to NNT 51) & NNT 96 (NNT 45 to NNH 210) \\
\hline Downstream testing $^{\star}$ & 43.6 & NNT 8 (NNT 3 to NNH 6) & NNH 4 (NNT 4 to NNH 2) & NNH 3 (NNH 7 to NNH 2) \\
\hline Any revascularisation & 3.0 & NNT 79 (NNT 58 to NNT 155) & NNT 40 (NNT 35 to NNT 97) & NNT 106 (NNT 72 to NNT 285) \\
\hline Myocardial infarction & 1.3 & NNH 133 (NNT 194 to NNH 25) & NNH 52 (NNT 110 to NNH 5) & NNH 196 (NNT 432 to NNH 58) \\
\hline \multicolumn{5}{|l|}{ Stable coronary artery disease $\S$} \\
\hline Invasive coronary angiography* & 12.2 & NNT 24 (NNT 16 to NNT 92) & NNT 20 (NNT 12 to NNH 73) & NNH 19 (NNT 92 to NNH 7) \\
\hline Downstream testing* & 21.2 & NNH 35 (NNT 14 to NNH 6) & NNH 11 (NNT 8 to NNH 2) & NNH 12 (NNT 11 to NNH 3) \\
\hline Any revascularisation & 6.2 & NNT 39 (NNT 28 to NNT 77) & NNT 106 (NNT 32 to NNH 40) & NNT 74 (NNT 36 to NNH 173) \\
\hline Myocardial infarction & 0.6 & NNH 480 (NNT 1289 to NNH 155) & NNH 102 (NNT 523 to NNH 19) & NNH 255 (NNT 16766 to NNH 95) \\
\hline Death & 1.5 & NNT 6767 (NNT 250 to NNH 189) & NNH 1129 (NNT 93 to NNH 24) & NNH 252 (NNT 217 to NNH 52) \\
\hline
\end{tabular}

*Estimated for procedures up to three months.

tRisk of events in patients receiving coronary computed tomographic angiography

¥Baseline risk is based on event rates of ACRIN-PA trial. ${ }^{37} 38$

$\S$ Baseline risk is based on event rates of PROMISE trial. ${ }^{35}$ 


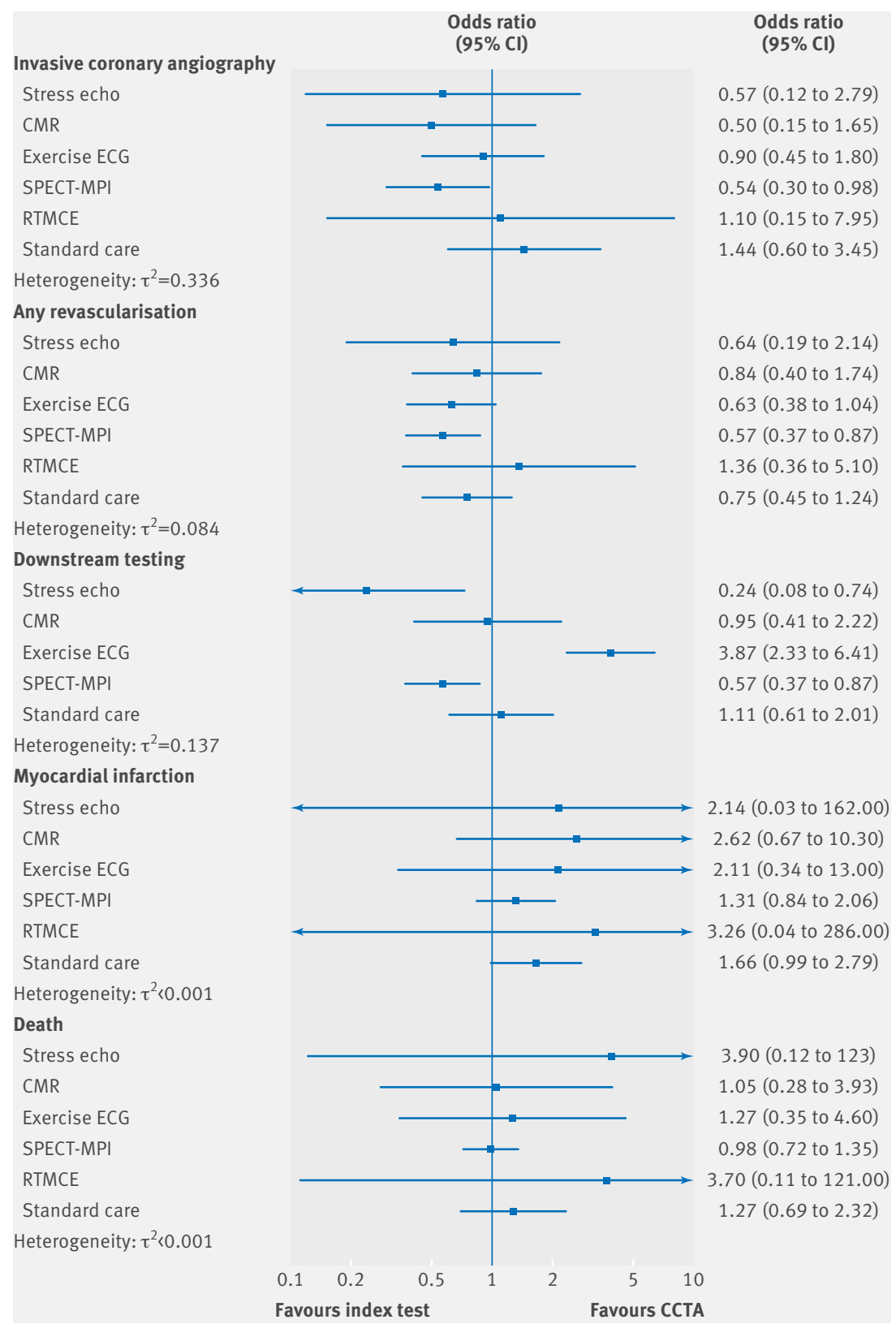

Fig 5 | Network meta-analysis effects of examined individual diagnostic strategies versus coronary computed tomographic angiography (anatomical testing), for study group of patients with stable coronary artery disease. Forest plot consider individual diagnostic strategies, as shown in figure $1 \mathrm{C}$. Index test indicates any diagnostic modality other than coronary computed tomographic angiography (anatomical testing). Real time myocardial contrast echocardiography was not included in the network meta-analysis for outcome of downstream testing because of unavailable data. $E C G=$ electrocardiogram; echo=echocardiography; $R T M C E=$ real time myocardial contrast echocardiography; SPECT-MPI=single photon emission computed tomographymyocardial perfusion imaging; CCTA=coronary computed tomographic angiography; $\mathrm{CMR}=$ cardiovascular magnetic resonance event of 1289 or more. The confidence interval also indicated that results were compatible with a harmful effect of functional testing, resulting in a number needed to harm to cause one event of 155 or more (table 2).

\section{Discussion \\ Main findings}

This study assesses the available evidence derived from diagnostic randomised controlled trials of strategies to detect coronary artery disease in a systematic and comprehensive way in two different clinical settings. Firstly, among patients with low risk acute coronary syndrome not required to undergo early invasive assessment, initial functional testing in terms of stress echocardiography or cardiovascular magnetic resonance was most strongly associated with a reduction of referrals for downstream invasive coronary angiography and revascularisation procedures, compared with anatomical testing using coronary CT angiography. No diagnostic strategy had an apparent effect on the subsequent risk of myocardial infarction, although estimates were imprecise.

Secondly, among patients with symptoms suggestive of stable coronary artery disease, no clear discrimination was seen across individual diagnostic strategies for the primary outcome of invasive coronary angiography referrals, mainly because of the limited number of trials contributing to each comparison. Stress echocardiography and single photon emission CTmyocardial perfusion imaging resulted in less overall downstream testing than coronary CT angiography, whereas exercise electrocardiograms required the most further downstream testing. After grouping of widely available functional tests, a functional testing approach yielded fewer referrals for invasive coronary angiography and subsequent revascularisations than anatomical testing. Again, estimates were imprecise for the outcome of myocardial infarction, and any differences could not be ruled out.

\section{Implications for patients with low risk acute coronary syndrome}

Among patients with suspected acute coronary syndrome without relevant electrocardiographic changes and negative biomarkers, several different non-invasive modalities have been tested in trials to detect clinically relevant coronary artery disease. In this clinical setting, coronary CT angiography has shown high sensitivity and negative predictive value, although the positive predictive value using invasive coronary angiography as reference standard has been shown to be moderate. ${ }^{7172}$

In our meta-analysis, a diagnostic strategy based on anatomical testing with use of coronary CT angiography was associated with increased referral rates for downstream invasive coronary angiography and revascularisation, some of which could have occurred in the absence of evidence of ischaemia. High sensitivity troponin assays, which were used in a minority of the included trials in our meta-analysis, are 


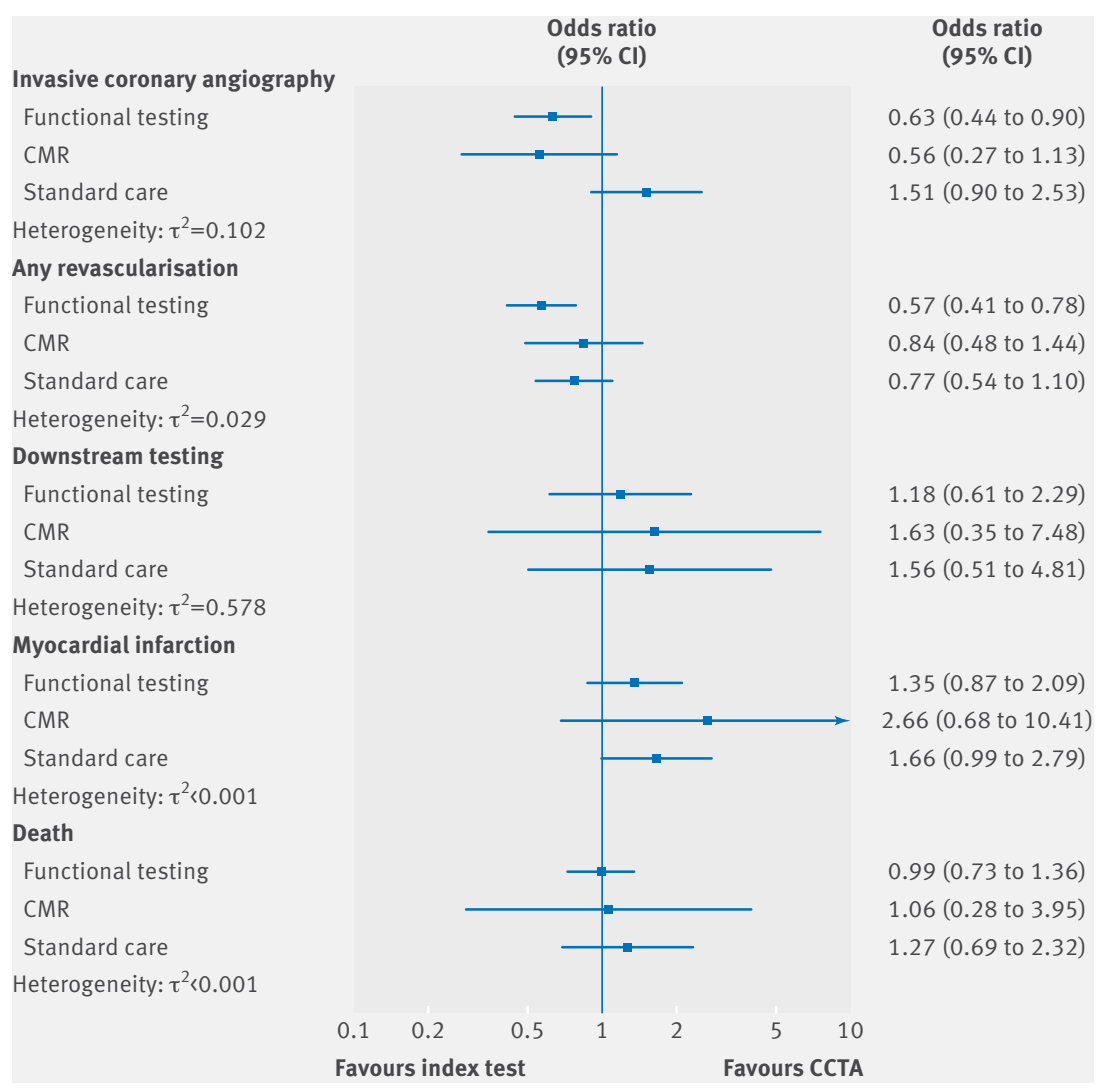

Fig 6 | Network meta-analysis effects of examined grouped diagnostic strategies versus coronary computed tomographic angiography (anatomical testing), for study group of patients with stable coronary artery disease. Forest plot considers grouping of functional tests, as shown in figure 1D. Index test indicates any diagnostic modality other than coronary computed tomographic angiography (anatomical testing). CCTA=coronary computed tomographic angiography; $C M R=$ cardiovascular magnetic resonance we found no difference in rates of overall downstream testing between anatomical and functional based strategies, while a standard of care approach was the strategy with the highest rates of downstream testing. This result could be attributed to the fact that doctors make the final decision to refer patients for further invasive testing on the basis of factors other than initial non-invasive imaging, such as clinical presentation, persistence of symptoms, repeated clinical encounters, and patient preference. ${ }^{74}$ A functional testing strategy might provide important cost benefits, owing to fewer referrals for invasive coronary angiography and revascularisation and lower radiation and contrast agent exposure while resulting in similar clinical outcomes. ${ }^{76}$ Such benefits could reduce healthcare expenditure in this common clinical scenario in appropriately selected patients with low risk acute coronary syndrome. However, the availability and rapid access to functional imaging modalities (such as stress echocardiography or cardiovascular magnetic resonance) in the acute coronary syndrome setting might be limited.

\section{Implications for patients with suspected stable coronary artery disease}

For outpatients with suspected stable angina, our comprehensive synthesis of diagnostic randomised controlled trials indicates that an initial strategy based on functional testing might be preferable, resulting in fewer referrals for invasive coronary angiography and revascularisation. However-as was the case for the group with acute coronary syndrome-our estimates for the risk of myocardial infarction and death were imprecise, and wide 95\% confidence intervals again cannot rule out relevant increases or reductions in the risk of myocardial infarction or death associated with functional testing.

Guidelines published in 2012 by the American College of Cardiology Foundation/American Heart Association recommend functional testing as the initial strategy. ${ }^{11}$ However, a concurrent use of functional and anatomical testing has been proposed on the basis of findings from two landmark trials (PROMISE ${ }^{35}$ and SCOT-HEART ${ }^{63}$ ) that evaluated the role of coronary CT angiography in patients with suspected stable coronary artery disease. ${ }^{77}$ Our results agree with these guidelines, but contradict the recently updated guidelines from the National Institute for Health and Care Excellence (NICE). The NICE guidelines advise a non-invasive anatomical approach (coronary CT angiography) as first line diagnostic strategy with subsequent functional testing only in case of inconclusive results of the initial diagnostic test, without considering the individual pretest probability of coronary artery disease. ${ }^{78}$

In a nationwide cohort study, ${ }^{79}$ Jorgensen and colleagues found a diagnostic approach based on non-invasive anatomical testing to be associated with modifications to cardiovascular related drug treatments, increased downstream invasive coronary testing and subsequent revascularisation, and a lower 
risk of myocardial infarction (hazard ratio 0.71, 95\% confidence interval 0.61 to 0.82 ) compared with functional testing. Similarly, a conventional metaanalysis including three trials in the corresponding analysis showed a borderline significant reduction of myocardial infarction with coronary CT angiography compared with a mixture of functional testing and standard care (odds ratio 0.69 (95\% confidence interval 0.49 to 0.98$)) .^{80}$

In our network meta-analysis, we found a statistically non-significant signal of a similar magnitude. Results in figure 6 correspond to an odds ratio of myocardial infarction of 0.74 favouring coronary CT angiography over functional testing (95\% confidence interval 0.48 to 1.15). However, our network meta-analysis made full use of all available evidence from 12 randomised trials comparing seven different diagnostic strategies within one analysis, appropriately quantifying the uncertainty of hard clinical outcomes associated with these strategies. Nevertheless, both the direction and magnitude of effects in our analysis are comparable with the large cohort study by Jorgensen and colleagues $^{79}$ and the conventional meta-analysis. ${ }^{80}$

A decrease in the risk of subsequent myocardial infarction related to an anatomical testing strategy is indeed possible and cannot be ruled out based on our results. However, whether intensification of medical treatment (primary or secondary prevention) or the increased rate of subsequent coronary revascularisation (or both) affect the prognosis of patients undergoing coronary CT angiography remains unknown. Finally, the baseline risk of myocardial infarction in the landmark PROMISE trial ${ }^{35}$ and the cohort study by Jorgensen and colleagues ${ }^{79}$ were low ( $0.6 \%$ and $0.8 \%$ for up to one month, respectively). This resulted in absolute risk differences between functional testing and coronary CT angiography of about $0.2 \%$, with a corresponding number needed to harm around 500 for this outcome (table 2), which is arguably irrelevant to raise safety concerns.

\section{Implications for clinicians, policymakers, and other} researchers

Diagnostic tests are critical components of an effective healthcare system. Diagnostic randomised controlled trials should become the default evaluation tool for new imaging modalities and clinical outcomes. ${ }^{81-83}$ Our systematic evaluation show that the low event rates have resulted in sample sizes of thousands of patients in recent trials but without allowing for a clear discrimination between the individual diagnostic strategies. Along the same lines, the use of broader clinical (composite) endpoints might be clinically meaningful in future trials. More importantly, the resulting networks of trials suggest that each technological innovation became the standard for subsequent future trials (that is, coronary CT angiography), although no clear advantage in terms of clinical outcomes had been shown compared with previous diagnostic strategies. Future adequately powered clinical trials should aim to clarify the differential effects on more broadly defined clinical outcomes (which could occur during longer follow-up periods), and subsequent use of hospital resources and cost effectiveness aspects of implemented strategies, which are representative of current clinical practice.

Currently, there is a broad range of non-invasive imaging modalities to investigate patients with suspected low risk acute coronary syndrome or stable coronary artery disease, with further studies required to determine how to best integrate these tests in the patient care pathway. Several parameters such as locally available technology and expertise, patient's preferences, and relevant contraindications for each test should be taken into account when deciding on the appropriate imaging modality. Any potential benefits of the applied diagnostic test should be carefully evaluated in the context of its risks (that is, radiation exposure for coronary CT or invasive coronary angiography).

\section{Study limitations}

This study had several limitations. Firstly, access to individual patient data that would have allowed us to identify potential differential effects in specific subgroups of patients was not available. Secondly, the low event rate of clinical outcomes (such as myocardial infarction and death) reflective of low risk populations and the sparse data for some comparisons (that is, those informed only by one trial) might have not provided enough power to clearly discriminate diagnostic strategies, especially within the stable coronary artery disease group. Thirdly, information on cost effectiveness as part of the downstream testing among the identified strategies was not given in most of the individual studies; therefore, we were not able to evaluate this aspect. Fourthly, the primary endpoints of invasive coronary angiography and revascularisation is partly attributed to physician judgment, which is not the case for the patient oriented outcomes of death and myocardial infarction. Finally, the standard of care, which was often a comparator in our study, has substantially evolved over the years. Earlier trials might not be directly relevant to present practice. However, the vast majority of the studies had been conducted in the past decade, and no differences during that period or compared with present practice are expected to have affect the results.

We thank Konstantinos Siontis (Division of Cardiovascular Medicine, University of Michigan, Ann Arbor) for his insightful comments on the protocol of the study, and the principal investigators of individual diagnostic randomised controlled trials who contributed to this study by providing additional unpublished data related to the outcomes of interest: Marisa Lubbers (Department of Cardiology, Erasmus Medical Centre, Rotterdam, Netherlands); Admir Dedic (Department of Cardiology, Erasmus University Medical Centre, Rotterdam, Netherlands); Roxy Senior (Department of Cardiovascular Medicine and Institute for Medical Research, Northwick Park Hospital, Harrow, UK; Centre for Health Informatics and Multi-Professional Education, University College London, London, UK; Cardiovascular, Biomedical Research Unit, Department of Cardiology, Royal Brompton Hospital, London, UK; Department of Cardiology, Imperial College London, London, UK); Pamela S Douglas (Duke University School of Medicine, Duke University Medical Center, Durham, USA); David Newby (British Heart Foundation Centre for Cardiovascular Science, University of Edinburgh, Edinburgh, UK) on behalf of the SCOTHEART Investigators; 
Jeffrey M Levsky (Department of Radiology, Montefiore Medical Center/Albert Einstein College of Medicine, Bronx, NY, USA); Peter McKavanagh (Cardiology Department, Ulster Hospital, South Eastern Health and Social Care Trust, Dundonald, Belfast, UK; Centre for Vision and Vascular Science, Queen's University Belfast, Royal Victoria Hospital Belfast, Belfast, UK); Jesper James Linde (Department of Cardiology, Hvidovre Hospital, University of Copenhagen, Denmark); Michel M Lièvre (Lyon 1 University, Louis Pradel Hospital, Bron, France); Frans I Th Wackers (Yale University School of Medicine, Section of Cardiovascular Medicine, New Haven, USA); Gaetano Nucifora (Azienda Ospedaliero-Universitaria di Udine, Udine, Italy).

Contributors: GCMS, DM, PJ, GS, and SW designed the study. BC built the search strategy. GCMS and DM screened the articles for eligibility and extracted the data. JPG provided additional unpublished data. GCMS, DM, AN, PJ, and GS performed the statistical analysis and interpreted the results. GCMS and SW wrote the first draft of the manuscript, with major contributions from JPG and PJ. All authors made revisions to the manuscript and have read and approved the final version. SW is the guarantor. All authors had full access to all of the data.

Funding: No funding was obtained for this study.

Competing interests: All authors have completed the ICMJE uniform disclosure form at www.icmie.org/coi disclosure.pdf and declare: no support from any organisation for the submitted work; JPG has received research grants to the institution from Boston Scientific, St Jude, and Philips Healthcare, and personal fees from Astra Zeneca, outside the submitted work; PJ has received research grants to the institution from Astra Zeneca, Biotronik, Biosensors International, Eli Lilly, and The Medicines Company outside the submitted work, and serves as unpaid member of the steering group of trials funded by Astra Zeneca, Biotronik, Biosensors, St Jude Medical, and The Medicines Company; PJ is a tier 1 Canada research chair in clinical epidemiology of chronic diseases, which was completed partly with funding from the Canada Research Chairs Program; GS is a Marie Skłodowska-Curie Fellow (grant MSCA-IF-703254); SW has received research grants to the institution from Bracco, Boston Scientific, Terumo, Abbott, St Jude, Edwards, Medtronic, and personal fees from Astra Zeneca, outside the submitted work; all other authors have nothing to declare; no other relationships or activities that could appear to have influenced the submitted work.

Ethical approval: No ethical approval was required.

Data sharing: No additional data are available.

Transparency: The corresponding author affirms that this manuscript is an honest, accurate, and transparent account of the study being reported; that no important aspects of the study have been omitted; and that any discrepancies from the study as planned (and, if relevant, registered) have been explained.

This is an Open Access article distributed in accordance with the Creative Commons Attribution Non Commercial (CC BY-NC 4.0) license, which permits others to distribute, remix, adapt, build upon this work non-commercially, and license their derivative works on different terms, provided the original work is properly cited and the use is non-commercial. See: http://creativecommons.org/licenses/ by-nc/4.0/

1 Amsterdam EA, Kirk ID, Bluemke DA, et al, American Heart Association Exercise, Cardiac Rehabilitation, and Prevention Committee of the Council on Clinical Cardiology, Council on Cardiovascular Nursing, and Interdisciplinary Council on Quality of Care and Outcomes Research. Testing of low-risk patients presenting to the emergency department with chest pain: a scientific statement from the American Heart Association. Circulation 2010;122: 1756-76. 10.1161/CIR.0b013e3181ec61df

2 Benjamin EJ, Blaha MJ, Chiuve SE, et al, American Heart Association Statistics Committee and Stroke Statistics Subcommittee. Heart Disease and Stroke Statistics-2017 Update: A Report From the American Heart Association [corrections in: Circulation 2017;135:e646; Circulation 2017:136:e196]. Circulation 2017:135:e146-603. 10.1161/ CIR.0000000000000485

3 Baxt WG, Shofer FS, Sites FD, Hollander JE. A neural network aid for the early diagnosis of cardiac ischemia in patients presenting to the emergency department with chest pain. Ann Emerg Med 2002;40:575-83.10.1067/mem.2002.129171

4 Selker HP, Beshansky JR, Griffith JL, et al. Use of the acute cardiac ischemia time-insensitive predictive instrument (ACl-TIPI) to assist with triage of patients with chest pain or other symptoms suggestive of acute cardiac ischemia. A multicenter, controlled clinical trial. Ann Intern Med 1998;129:845-55. 10.7326/0003-4819-12911 Part 1-199812010-00002
5 Damen JA, Hooft L, Schuit E, et al. Prediction models for cardiovascular disease risk in the general population: systematic review. BMJ 2016:353:i2416. 10.1136/bmj.i2416

6 Than M, Cullen L, Reid CM, et al. A 2-h diagnostic protocol to assess patients with chest pain symptoms in the Asia-Pacific region (ASPECT): a prospective observational validation study. Lancet 2011;377:1077-84. 10.1016/S0140-6736(11)60310-3

7 Reichlin T, Hochholzer W, Bassetti S, et al. Early diagnosis of myocardial infarction with sensitive cardiac troponin assays. N Engl/ Med 2009;361:858-67. 10.1056/NEJMoa0900428

8 Keller T, Zeller T, Peetz D, et al. Sensitive troponin I assay in early diagnosis of acute myocardial infarction. $N$ Engl/ Med 2009;361:868-77. 10.1056/NEJMoa0903515

9 Sekhri N, Feder GS, Junghans C, Hemingway H, Timmis AD. How effective are rapid access chest pain clinics? Prognosis of incident angina and non-cardiac chest pain in 8762 consecutive patients. Heart 2007;93:458-63. 10.1136/hrt.2006.090894

10 Jordan KP, Timmis A, Croft P, et al. Prognosis of undiagnosed chest pain: linked electronic health record cohort study. BMJ 2017;357:j1194. 10.1136/bmj.j1194

11 Fihn SD, Gardin JM, Abrams J, et al, American College of Cardiology Foundation/American Heart Association Task Force. 2012 ACCF/ AHA/ACP/AATS/PCNA/SCAI/STS guideline for the diagnosis and management of patients with stable ischemic heart disease: a report of the American College of Cardiology Foundation/ American Heart Association task force on practice guidelines, and the American College of Physicians, American Association for Thoracic Surgery, Preventive Cardiovascular Nurses Association, Society for Cardiovascular Angiography and Interventions, and Society of Thoracic Surgeons [correction in: Circulation 2014;129:e463]. Circulation 2012;126:e354-471. 10.1161/ CIR.0b013e318277d6a0

12 Patel MR, Peterson ED, Dai D, et al. Low diagnostic yield of elective coronary angiography. N Engl/ Med 2010;362:886-95. 10.1056/ NEJMoa0907272

13 de Azevedo CF, Hadlich MS, Bezerra SG, et al. Prognostic value of CT angiography in patients with inconclusive functional stress tests. JACC Cardiovasc Imaging 2011;4:740-51. 10.1016/j.jcmg.2011.02.017

14 Poon M, Cortegiano M, Abramowicz AJ, et al. Associations between routine coronary computed tomographic angiography and reduced unnecessary hospital admissions, length of stay, recidivism rates, and invasive coronary angiography in the emergency department triage of chest pain. J Am Coll Cardiol 2013;62:543-52. 10.1016/j. jacc.2013.04.040

15 National Institute for Health Research. PROSPERO International prospective register of systematic reviews. Siontis G, Mavridis D, Siontis K, et al. Diagnostic strategies for patients with suspected coronary artery disease: a network meta-analysis of diagnostic randomized controlled trials. www.crd.york.ac.uk/PROSPERO/ display_record.php?ID=CRD42016049442.

16 Chaimani A, Caldwell DM, Li T, Higgins JP, Salanti G. Additional considerations are required when preparing a protocol for a systematic review with multiple interventions. / Clin Epidemiol 2017;83:65-74.

17 Salanti G. Indirect and mixed-treatment comparison, network, or multiple-treatments meta-analysis: many names, many benefits, many concerns for the next generation evidence synthesis tool. Res Synth Methods 2012;3:80-97. 10.1002/jrsm.1037

18 Neglia D, Rovai D, Caselli C, et al, EVINCI Study Investigators. Detection of significant coronary artery disease by noninvasive anatomical and functional imaging. Circ Cardiovasc Imaging 2015;8:e002179. 10.1161/CIRCIMAGING.114.002179

19 Arbab-Zadeh A, Di Carli MF, Cerci R, et al. Accuracy of Computed Tomographic Angiography and Single-Photon Emission Computed Tomography-Acquired Myocardial Perfusion Imaging for the Diagnosis of Coronary Artery Disease. Circ Cardiovasc Imaging 2015;8:e003533. 10.1161/CIRCIMAGING.115.003533

20 Higgins JP, Altman DG, Gøtzsche PC, Cochrane Bias Methods GroupCochrane Statistical Methods Group. The Cochrane Collaboration's tool for assessing risk of bias in randomised trials. BMJ 2011;343:d5928. 10.1136/bmj.d5928

21 Cipriani A, Higgins JP, Geddes JR, Salanti G. Conceptual and technical challenges in network meta-analysis. Ann Intern Med 2013:159: 130-7. 10.7326/0003-4819-159-2-201307160-00008

22 Lu G, Ades AE. Combination of direct and indirect evidence in mixed treatment comparisons. Stat Med 2004:23:3105-24. 10.1002/ sim. 1875

23 DerSimonian R, Laird N. Meta-analysis in clinical trials revisited. Contemp Clin Trials 2015;45(Pt A):139-45. 10.1016/j. cct.2015.09.002

24 Nikolakopoulou A, Mavridis D, Salanti G. Demystifying fixed and random effects meta-analysis. Evid Based Ment Health 2014;17: 53-7. 10.1136/eb-2014-101795

25 Mavridis D, Giannatsi M, Cipriani A, Salanti G. A primer on network meta-analysis with emphasis on mental health. Evid Based Ment Health 2015:18:40-6. 10.1136/eb-2015-102088 
26 Siontis GC, Stefanini GG, Mavridis D, et al. Percutaneous coronary interventional strategies for treatment of in-stent restenosis: a network meta-analysis. Lancet 2015;386:655-64. 10.1016/S01406736(15)60657-2

27 Efthimiou O, Debray TP, van Valkenhoef G, et al, GetReal Methods Review Group. GetReal in network meta-analysis: a review of the methodology. Res Synth Methods 2016;7:236-63. 10.1002/ jrsm.1195

28 Salanti G, Ades AE, loannidis JP. Graphical methods and numerical summaries for presenting results from multiple-treatment metaanalysis: an overview and tutorial. / Clin Epidemiol 2011;64:163-71. 10.1016/j.jclinepi.2010.03.016

29 Turner RM, Davey J, Clarke MJ, Thompson SG, Higgins JP. Predicting the extent of heterogeneity in meta-analysis, using empirical data from the Cochrane Database of Systematic Reviews. Int Epidemiol 2012:41:818-27.10.1093/ije/dys041

30 da Costa BR, Juni P. Systematic reviews and meta-analyses of randomized trials: principles and pitfalls. Eur Heart J 2014;35: 3336-45. 10.1093/eurheartj/ehu424

31 Chaimani A, Higgins JP, Mavridis D, Spyridonos P, Salanti G. Graphical tools for network meta-analysis in STATA. PLoS One 2013;8:e76654 10.1371/journal.pone.0076654

32 Dias S, Welton NJ, Caldwell DM, Ades AE. Checking consistency in mixed treatment comparison meta-analysis. Stat Med 2010;29: 932-44. 10.1002/sim.3767

33 Higgins JP, Jackson D, Barrett JK, Lu G, Ades AE, White IR. Consistency and inconsistency in network meta-analysis: concepts and models for multi-arm studies. Res Synth Methods 2012;3:98-110. 10.1002/ jrsm.1044

34 Lubbers M, Dedic A, Coenen A, et al. Calcium imaging and selective computed tomography angiography in comparison to functional testing for suspected coronary artery disease: the multicentre, randomized CRESCENT trial. Eur Heart / 2016:37:1232-43. 10.1093/eurheartj/ehv700

35 Douglas PS, Hoffmann U, Patel MR, et al, PROMISE Investigators. Outcomes of anatomical versus functional testing for coronary artery disease. N Engl J Med 2015;372:1291-300. 10.1056/ NEJMoa1415516

36 Del Giovane C, Vacchi L, Mavridis D, Filippini G, Salanti G. Network meta-analysis models to account for variability in treatment definitions: application to dose effects. Stat Med 2013;32:25-39. $10.1002 / \operatorname{sim} .5512$

37 Litt HI, Gatsonis C, Snyder B, et al. CT angiography for safe discharge of patients with possible acute coronary syndromes. $\mathrm{N}$ Engl J Med 2012;366:1393-403. 10.1056/NEJMoa1201163

38 Hollander JE, Gatsonis C, Greco EM, et al. Coronary Computed Tomography Angiography Versus Traditiona Care: Comparison of One-Year Outcomes and Resource Use. Ann Emerg Med 2016;67:460-468.e1. 10.1016/j. annemergmed.2015.09.014

39 The Stata Journal. www.stata-journal.com/article.html?article=st0410

40 CINeMA: Confidence in Network Meta-Analysis [software]. Institute of Social and Preventive Medicine, University of Bern, 2017. cinema. ispm.ch.

41 Dedic A, Lubbers MM, Schaap J, et al. Coronary CT Angiography for Suspected ACS in the Era of High-Sensitivity Troponins: Randomized Multicenter Study. J Am Coll Cardiol 2016;67:16-26. 10.1016/j. jacc.2015.10.045

42 Levsky JM, Spevack DM, Travin MI, et al. Coronary Computed Tomography Angiography Versus Radionuclide Myocardial Perfusion Imaging in Patients With Chest Pain Admitted to Telemetry: A Randomized Trial. Ann Intern Med 2015;163:174-83. 10.7326/ M14-2948

43 Hamilton-Craig C, Fifoot A, Hansen M, et al. Diagnostic performance and cost of CT angiography versus stress ECG--a randomized prospective study of suspected acute coronary syndrome chest pain in the emergency department (CT-COMPARE). Int ) Cardiol 2014;177:867-73.10.1016/j.ijcard.2014.10.090

44 Linde JJ, Kofoed KF, Sørgaard M, et al. Cardiac computed tomography guided treatment strategy in patients with recent acute-onset chest pain: results from the randomised, controlled trial: CArdiac CT in the treatment of acute CHest pain (CATCH). Int J Cardiol 2013;168: 5257-62. 10.1016/j.jicard.2013.08.020

45 Linde JJ, Hove JD, Sørgaard M, et al. Long-Term Clinical Impact of Coronary CT Angiography in Patients With Recent Acute-Onset Chest Pain: The Randomized Controlled CATCH Trial. JACC CardiovasC Imaging 2015;8:1404-13. 10.1016/j.jcmg.2015.07.015

$46 \mathrm{Lim}$ SH, Anantharaman V, Sundram F, et al. Stress myocardial perfusion imaging for the evaluation and triage of chest pain in the emergency department: a randomized controlled trial. / Nuc Cardiol 2013;20:1002-12. 10.1007/s12350-013-9736-9

47 Miller CD, Case LD, Little WC, et al. Stress CMR reduces revascularization, hospital readmission, and recurrent cardiac testing in intermediate-risk patients with acute chest pain. JACC CardiovasC Imaging 2013;6:785-94. 10.1016/j.jcmg.2012.11.022
48 Hoffmann U, Truong QA, Schoenfeld DA, et al, ROMICAT-II Investigators. Coronary CT angiography versus standard evaluation in acute chest pain. N Engl J Med 2012;367:299-308. 10.1056/ NEJMoa1201161

49 Goldstein JA, Chinnaiyan KM, Abidov A, et al, CT-STAT Investigators. The CT-STAT (Coronary Computed Tomographic Angiography for Systematic Triage of Acute Chest Pain Patients to Treatment) trial. Am Coll Cardiol 2011;58:1414-22. 10.1016/j.jacc.2011.03.068

50 Miller AH, Pepe PE, Peshock R, et al. Is coronary computed tomography angiography a resource sparing strategy in the risk stratification and evaluation of acute chest pain? Results of a randomized controlled trial. Acad Emerg Med 2011;18:458-67. 10.1111/j.1553-2712.2011.01066.x

51 Miller CD, Hwang W, Hoekstra JW, et al. Stress cardiac magnetic resonance imaging with observation unit care reduces cost for patients with emergent chest pain: a randomized trial. Ann Emerg Med 2010;56:209-219.e2. 10.1016/j.annemergmed.2010.04.009

52 Miller CD, Hwang W, Case D, et al. Stress CMR imaging observation unit in the emergency department reduces 1-year medical care costs in patients with acute chest pain: a randomized study for comparison with inpatient care. JACC Cardiovasc Imaging 2011;4:862-70. 10.1016/j.jcmg.2011.04.016

53 Nucifora G, Badano LP, Sarraf-Zadegan N, et al. Effect on quality of life of different accelerated diagnostic protocols for management of patients presenting to the emergency department with acute chest pain. Am / Cardiol 2009;103:592-7. 10.1016/j. amjcard.2008.10.030

54 Chang SA, Choi SI, Choi EK, et al. Usefulness of 64-slice multidetecto computed tomography as an initial diagnostic approach in patients with acute chest pain. Am Heart J 2008;156:375-83. 10.1016/j. ahj.2008.03.016

55 Goldstein JA, Gallagher MJ, O’Neill WW, Ross MA, O’Neil BJ, Raff GL. A randomized controlled trial of multi-slice coronary computed tomography for evaluation of acute chest pain. J Am Coll Cardiol 2007;49:863-71. 10.1016/j.jacc.2006.08.064

56 Jeetley P, Burden L, Stoykova B, Senior R. Clinical and economic impact of stress echocardiography compared with exercise electrocardiography in patients with suspected acute coronary syndrome but negative troponin: a prospective randomized controlled study. Eur Heart J 2007;28:204-11. 10.1093/eurheartj/ ehl 1444

57 Nucifora G, Badano LP, Sarraf-Zadegan N, et al. Comparison of early dobutamine stress echocardiography and exercise electrocardiographic testing for management of patients presenting to the emergency department with chest pain. Am J Cardiol 2007;100:1068-73. 10.1016/j.amjcard.2007.05.027

58 Jeetley P, Burden L, Senior R. Stress echocardiography is superior to exercise ECG in the risk stratification of patients presenting with acute chest pain with negative Troponin. Eur J Echocardiogr 2006; 155-64. 10.1016/j.euje.2005.05.002

59 Udelson JE, Beshansky JR, Ballin DS, et al. Myocardial perfusion imaging for evaluation and triage of patients with suspected acute cardiac ischemia: a randomized controlled trial. JAMA 2002;288:2693-700. 10.1001/jama.288.21.2693

60 Karthikeyan G, Guzic Salobir B, Jug B, et al. Functional compared to anatomical imaging in the initial evaluation of patients with suspected coronary artery disease: An international, multi-center, randomized controlled trial (IAEA-SPECT/CTA study). J Nucl Cardiol 2017:24:507-17. 10.1007/s12350-016-0664-3

61 Greenwood IP, Ripley DP, Berry C, et al, CE-MARC 2 Investigators. Effect of Care Guided by Cardiovascular Magnetic Resonance, Myocardial Perfusion Scintigraphy, or NICE Guidelines on Subsequent Unnecessary Angiography Rates: The CE-MARC 2 Randomized Clinical Trial. JAMA 2016;316:1051-60. 10.1001/ jama.2016.12680

62 Zacharias K, Ahmed A, Shah BN et al. Relative clinical and economic impact of exercise echocardiography vs. exercise electrocardiography, as first line investigation in patients without known coronary artery disease and new stable angina: a randomized prospective study. Eur Heart / Cardiovasc Imaging 2017;18: 195-202. 10.1093/ehjci/jew049

63 SCOT-HEART investigators. CT coronary angiography in patients with suspected angina due to coronary heart disease (SCOT-HEART): an open-label, parallel-group, multicentre trial. Lancet 2015;385: 2383-91. 10.1016/S0140-6736(15)60291-4

64 Williams MC, Hunter A, Shah ASV, et al, SCOT-HEART Investigators. Use of Coronary Computed Tomographic Angiography to Guide Management of Patients With Coronary Disease. J Am Coll Cardiol 2016;67:1759-68. 10.1016/j. jacc.2016.02.026

65 Laiq Z, Smith LM, Xie F, Chamsi-Pasha M, Porter TR. Differences in patient outcomes after conventional versus real time perfusion stress echocardiography in men versus women: a prospective randomised trial. Heart 2015;101:559-64. 10.1136/ heartjnl-2014-306869 
66 McKavanagh P, Lusk L, Ball PA, et al. A comparison of cardiac computerized tomography and exercise stress electrocardiogram test for the investigation of stable chest pain: the clinical results of the CAPP randomized prospective trial. Eur Heart J Cardiovas C Imaging 2015;16:441-8. 10.1093/ehjci/jeu284

67 Porter TR, Smith LM, Wu J, et al. Patient outcome following 2 different stress imaging approaches: a prospective randomized comparison. J Am Coll Cardiol 2013;61:2446-55. 10.1016/j. jacc.2013.04.019

68 Min JK, Koduru S, Dunning AM, et al. Coronary CT angiography versus myocardial perfusion imaging for near-term quality of life, cost and radiation exposure: a prospective multicenter randomized pilot trial. / Cardiovasc Comput Tomogr 2012;6:274-83. 10.1016/j. jcct.2012.06.002

69 Shaw LJ, Mieres JH, Hendel RH, et al, WOMEN Trial Investigators. Comparative effectiveness of exercise electrocardiography with or without myocardial perfusion single photon emission computed tomography in women with suspected coronary artery disease: results from the What Is the Optimal Method for Ischemia Evaluation in Women (WOMEN) trial. Circulation 2011;124:1239-49. 10.1161/ CIRCULATIONAHA.111.029660

70 Sabharwal NK, Stoykova B, Taneja AK, Lahiri A. A randomized tria of exercise treadmill ECG versus stress SPECT myocardial perfusion imaging as an initial diagnostic strategy in stable patients with chest pain and suspected CAD: cost analysis. I Nucl Cardiol 2007;14 174-86. 10.1016/j.nuclcard.2006.12.324

71 Rubinshtein R, Halon DA, Gaspar T, et al. Usefulness of 64-slice cardiac computed tomographic angiography for diagnosing acute coronary syndromes and predicting clinical outcome in emergency department patients with chest pain of uncertain origin. Circulation 2007;115:1762-8. 10.1161/ CIRCULATIONAHA.106.618389

72 Gallagher MJ, Ross MA, Raff GL, Goldstein JA, O’Neill WW, O’Neil B. The diagnostic accuracy of 64-slice computed tomographycoronary angiography compared with stress nuclear imaging in emergency department low-risk chest pain patients. Ann Emerg Med 2007; 49:125-36. 10.1016/j.annemergmed.2006.06.043

73 Shah ASV, Sandoval Y, Noaman A, et al. Patient selection for high sensitivity cardiac troponin testing and diagnosis of myocardial infarction: prospective cohort study [correction in: BMJ 2018;360:k495]. BMJ 2017;359:j4788. 10.1136/bmj.j4788

74 Engel J, Poldervaart JM, van der Wulp I, et al. Selecting patients with non-ST-elevation acute coronary syndrome for coronary angiography: a nationwide clinical vignette study in the Netherlands. BMJ Open 2017;7:e011213. 10.1136/bmjopen-2016-011213

75 Fanaroff AC, Rymer JA, Goldstein SA, Simel DL, Newby LK. Does This Patient With Chest Pain Have Acute Coronary Syndrome? The Rational Clinical Examination Systematic Review. IAMA 2015:314:1955-65. 10.1001/jama.2015.12735

76 Ladapo JA, Jaffer FA, Hoffmann U, et al. Clinical outcomes and cost-effectiveness of coronary computed tomography angiography in the evaluation of patients with chest pain. J Am Coll Cardiol 2009.54:2409-22.10.1016/j.jacc 2009.10.012

77 Fordyce CB, Newby DE, Douglas PS. Diagnostic Strategies for the Evaluation of Chest Pain: Clinical Implications From SCOT-HEART and PROMISE. J Am Coll Cardiol 2016;67:843-52. 10.1016/j. jacc.2015.11.055
78 Chest pain of recent onset: assessment and diagnosis. Clinical guideline. https://www.nice.org.uk/guidance/cg95

79 Jørgensen ME, Andersson C, Nørgaard BL, et al. Functional Testing or Coronary Computed Tomography Angiography in Patients With Stable Coronary Artery Disease. J Am Coll Cardiol 2017;69:1761-70. 10.1016/j.jacc.2017.01.046

80 Bittencourt MS, Hulten EA, Murthy VL, et al. Clinical Outcomes After Evaluation of Stable Chest Pain by Coronary Computed Tomographic Angiography Versus Usual Care: A Meta-Analysis. Circ Cardiovasc Imaging 2016;9:e004419. 10.1161/CIRCIMAGING.115.004419

81 Lord SJ, Irwig L, Simes RJ. When is measuring sensitivity and specificity sufficient to evaluate a diagnostic test, and when do we need randomized trials? Ann Intern Med 2006;144:850-5. 10.7326/0003-4819-144-11-200606060-00011

82 Siontis KC, Siontis GC, Contopoulos-Ioannidis DG, Ioannidis JP. Diagnostic tests often fail to lead to changes in patient outcomes. Clin Epidemiol 2014;67:612-21. 10.1016/j.jclinepi.2013.12.008

83 Ferrante di Ruffano L, Hyde CJ, McCaffery KJ, Bossuyt PM, Deeks JJ. Assessing the value of diagnostic tests: a framework for designing and evaluating trials. BMJ 2012;344:e686. 10.1136/bmj.e686

Web appendix 1: Systematic review searching record Web appendix 2: D-RCTs selection flow-chart Web appendix 3: Diagnostic accuracy of non-invasive diagnostic modalities

Web appendix 4: Abbreviations

Web appendix 5: (A) Eligible diagnostic randomized controlled trials and study populations' characteristics. (B) Assessed outcomes and number of events

Web appendix 6: (A) Risk of bias assessment. (B) Lowrisk acute coronary syndrome - Risk of bias table. (C) Low-risk acute coronary syndrome - Functional testing grouped - Risk of bias table. (D) Stable coronary artery disease - Risk of bias table. (E) Stable coronary artery disease - Functional testing grouped - Risk of bias table.

Web appendix 7: Low-risk acute coronary syndrome Web appendix 8: Low-risk acute coronary syndrome Functional testing grouped

Web appendix 9: Stable coronary artery disease Web appendix 10: Stable coronary artery disease Functional testing grouped

Web appendix 11: Stable coronary artery disease Sensitivity analysis without including CRESCENT and PROMISE trials 